\title{
Xenopus Vocalizations Are Controlled by a Sexually Differentiated Hindbrain Central Pattern Generator
}

\author{
Heather J. Rhodes, Heather J. Yu, and Ayako Yamaguchi \\ Department of Biology, Boston University, Boston, Massachusetts 02215
}

\begin{abstract}
Male and female African clawed frogs (Xenopus laevis) produce rhythmic, sexually distinct vocalizations as part of courtship and mating. We found that Xenopus vocal behavior is governed by a sexually dimorphic central pattern generator (CPG) and that fictive vocalizations can be elicited from an in vitro brain preparation by application of serotonin or by electrical stimulation of a premotor nucleus. Male brains produced fictive vocal patterns representing two calls commonly produced by males in vivo (advertisement and amplectant call), as well as one call pattern (release call) that is common for juvenile males and females in vivo but rare for adult males. Female brains also produced fictive release call. The production of male calls is androgen dependent in Xenopus; to test the effects of androgens on the CPG, we examined fictive calling in the brains of testosterone-treated females. Both fictive male advertisement call and release call were produced. This suggests that all Xenopus possess a sexually undifferentiated pattern generator for release call. Androgen exposure leads to a gain-of-function, allowing the production of male-specific call types without prohibiting the production of the undifferentiated call pattern. We also demonstrate that the CPG is located in the brainstem and seems to rely on the same nuclei in both males and females. Finally, we identified endogenous serotonergic inputs to both the premotor and motor nuclei in the brainstem that may regulate vocal activity in vivo.
\end{abstract}

Key words: frog; central pattern generator; rhythm; sex difference; serotonin; vocalization

\section{Introduction}

The study of central pattern generators (CPGs) has proven an effective way to elucidate neural mechanisms of behavior. CPGs, groups of neurons that generate rhythmic output without sensory input, govern rhythmic behaviors, such as locomotion and respiration, as well as sexually dimorphic behaviors, such as insect oviposition and fish vocalization (Wyman, 1976; Thompson, 1986; Bass and Baker, 1990; Grillner et al., 1998; Marder and Bucher, 2001).

Xenopus produce sex-specific vocalizations for social communication (Kelley and Tobias, 1998). All vocalizations are rhythmic and stereotyped, consisting of "clicks" in sex- and call-specific temporal patterns. Simple peripheral sound production machinery makes this species an excellent model for studying neural mechanisms of behavior. A compound action potential (CAP) produced by laryngeal motoneurons in the brainstem leads to the contraction of a pair of laryngeal muscles, producing one click of sound (Yager, 1992; Yamaguchi and Kelley, 2000). Although the rhythmic activation of motoneurons determines call type, it is not known how the motoneuron rhythm is established or how pattern generation differs in males and females. Like most sexu-

Received 0ct. 30, 2006; revised Dec. 28, 2006; accepted Jan. 8, 2007

This work was supported by National Institute of Neurological Disorders and Stroke Grant R01 NS048834, startup funds provided by Boston University, a Clare Boothe Luce Professorship (A.Y.), and National Research Service Award Postdoctoral Fellowship NS054391 (H.J.R.). We thank Shane Smith for technical assistance and Erik Zornik, Matt Wachowiak, Don Katz, and our anonymous reviewers for helpful comments on this manuscript.

Correspondence should be addressed to H. J. Rhodes, Department of Biology, Boston University, 5 Cummington Street, Boston, MA 02215. E-mail: hjc@bu.edu.

DOI:10.1523/JNEUROSCI.4720-06.2007

Copyright $\odot 2007$ Society for Neuroscience $\quad$ 0270-6474/07/271485-12\$15.00/0 ally dimorphic behaviors, Xenopus vocalizations are hormone dependent; male-type calling is androgen dependent and can be observed in testosterone-treated adult females (Hannigan and Kelley, 1986; Potter et al., 2005).

The anatomy of the central vocal-motor pathway is known in Xenopus (Wetzel et al., 1985; Brahic and Kelley, 2003; Zornik and Kelley, 2007). Laryngeal motoneurons are located in nucleus IX-X and have robust, reciprocal connections with the primary premotor nucleus, the dorsal tegmental area of medulla (DTAM) (homologous to the pretrigeminal nucleus). The dorsal raphe nucleus and the reticular formation, also in the brainstem, project to DTAM and nucleus IX-X. The premotor nuclei receive input from telencephalic and diencephalic nuclei, including the ventral striatum, anterior preoptic area, infundibular nuclei, and thalamus.

We hypothesized that Xenopus vocalizations are controlled by a sex-specific CPG that can be masculinized by testosterone exposure. We tested this hypothesis by applying serotonin (5-HT), a neurotransmitter that commonly activates CPGs (Jacobs and Fornal, 1997), to male, female, and testosterone-treated female (T-female) Xenopus brains in vitro. We found that 5-HT elicited sex-specific fictive vocal patterns, confirming that a CPG governs Xenopus vocal behavior. Male call patterns were androgen dependent, observed only in male and T-female brains, whereas a call pattern that is predominantly produced by females in vivo was seen in all groups, suggesting the existence of a sexually undifferentiated CPG that remains intact after androgens modify the vocal system enabling male-specific patterns.

Next, we investigated which nuclei in the vocal-motor circuit underlie pattern generation and found that the CPG was re- 
stricted to the hindbrain and that DTAM was necessary for 5-HTinduced fictive vocalizations in both sexes. Furthermore, local stimulation of DTAM evoked fictive vocal patterns, supporting DTAM as a candidate CPG. We found serotonergic projections from dorsal raphe neurons to motor and premotor nuclei in both sexes, suggesting a role for endogenous serotonin in vocal production. In addition to illuminating CPG structure, these findings suggest that homologous neural circuits are capable of producing sexually distinct activity patterns.

\section{Materials and Methods}

Animals. Thirty-four sexually mature males (snout-vent length, 6.5-9.0 $\mathrm{cm}$; mass, 32-75 g) and 17 sexually mature females (7.3-9.7 cm; 38-98 g) were purchased from NASCO (Fort Atkinson, WI). The animals were kept in glass aquaria on a $12 \mathrm{~h}$ light/dark cycle at room temperature. All experimental procedures were approved by the Boston University Institutional Animal Care and Use Committee and were performed in compliance with guidelines published by the National Institutes of Health.

Gonadectomy and testosterone implants. Six females were anesthetized with ethyl 3-amino benzoate methanesulfonic acid (MS-222) (Sigma, St. Louis, MO) and ovariectomized by removing ovaries and fat bodies using a cauterizer through a small incision in the abdomen. Four of the females were implanted with testosterone-filled (4-androsten-17 $\beta$-ol-3-one) (Sigma) SILASTIC tubes $(2.16 \mathrm{~mm}$ outer diameter $\times 1.02 \mathrm{~mm}$ inner diameter; $0.5 \mathrm{mg} / \mathrm{g}$ body weight), and the remaining two females were implanted with empty SILASTIC tubes into the ventral lymph sacs. One $\mathrm{T}$-female and two control females were killed 5 months after surgery, and three T-females were killed 8 weeks after surgery to be used for in vitro nerve recording experiments. Examination at the time of experiment revealed that the ovariectomy was complete, testosterone capsules were intact, and the larynx morphology was masculinized as described previously (Hannigan and Kelley, 1986; Potter et al., 2005). T-females are known to produce masculinized vocalizations and show masculinized motoneuron morphology after 8 weeks of testosterone exposure, whereas control females maintain their female-typical vocalizations (Hannigan and Kelley, 1986; Potter et al., 2005).

In vivo nerve recordings. Methods of recording nerve activity from awake vocalizing Xenopus have been described previously (Yamaguchi and Kelley, 2000). Briefly, a bipolar hook electrode made of Tefloncoated silver wire was surgically implanted onto the laryngeal nerve of an anesthetized adult male frog. The animal was placed into an aquarium equipped with a hydrophone while "tethered" to the differential amplifier, and the vocalizations and nerve activity were recorded simultaneously. Collection and analysis of vocal and nerve recording data were as described below.

Vocal recordings. Ten adult males were induced to advertisement calling by injection of $600 \mathrm{IU}$ of human chorionic gonadotropin (HCG) (Sigma) subcutaneously. HCG injection promotes advertisement call production but does not alter the structure of the call produced. Animals were placed alone in a $12 \mathrm{~L}$ tank with a hydrophone (H2; Aquarian Audio Products, Anacortes, WA) suspended in the water. Vocalizations were recorded overnight, in the dark, using a sound-activated recording system (Syrinx software, www.syrinxpc.com; John Burt, University of Washington, Seattle, WA). In vitro nerve recordings (below) were performed the following day ( $<24 \mathrm{~h}$ after HCG injection).

Vocalizations were also recorded from three of the T-females (the three killed at 8 weeks after the operation) and four intact females (those used for transection experiments). These animals were not given HCG; instead, a second animal was placed in the tank to stimulate calling. Both males and females were used as stimulus animals for T-females, because it is known that $\mathrm{T}$-female vocalizations are dependent on social context (Potter et al., 2005); intact females were recorded in the presence of a male. Female-like calls from the male/T-female pairing and male-like calls from the female/T-female pairing were determined to have been produced by the T-female and were used for analysis (Potter et al., 2005); all other calls were excluded.

In vitro nerve recordings of 5-HT-induced activity. Twelve adult males (including the 10 used for vocal recordings), six adult females, four testosterone-implanted females, and two control-implanted females were used for in vitro nerve recordings of 5-HT-induced activity. Frogs were anesthetized with MS-222 ( $0.15 \mathrm{mg} / \mathrm{g}$ body weight $)$ injected subcutaneously (Sigma) and decapitated. The brain was dissected away from the skull while submerged in oxygenated $\left(99 \% \mathrm{O}_{2} / 1 \% \mathrm{CO}_{2}\right)$ ice-cold saline composed of the following (in $\mathrm{mm}$ ): $96 \mathrm{NaCl}, 20 \mathrm{NaHCO}_{3}, 2$ $\mathrm{CaCl}_{2}, 2 \mathrm{KCl}, 0.5 \mathrm{MgCl}_{2}, 10 \mathrm{HEPES}$, and 11 glucose, $\mathrm{pH}$ 7.8. The brain was then transferred to a recording chamber in which it was continually superfused with fresh oxygenated saline and allowed to return to room temperature over the course of $\sim 1 \mathrm{~h}$.

A suction electrode was placed on the most caudal (fourth) rootlet of the nerve IX-X to record population activity as CAPs; this nerve root contains the axons of the laryngeal and glottal motoneurons (Simpson et al., 1986). The population activity recorded with the suction electrode was amplified [Warner Instruments (Hamden, CT) differential amplifier DP-301 or A-M Systems (Carlsborg, WA) differential amplifier 1700], high-pass filtered $(1 \mathrm{~Hz}$ ), digitized at $10 \mathrm{kHz}$ (Digidata 1322A; Molecular Devices, Sunnyvale, CA), and recorded on a personal computer using AxoScope software (Molecular Devices).

5-HT was applied by replacing half of the saline in the recording chamber with $60 \mu \mathrm{M}$ 5-HT dissolved in oxygenated saline to achieve the final concentration of $30 \mu \mathrm{M}$. 5-HT application took $20 \mathrm{~s}$ or less, after which 5-HT remained on the brain for 2-10 min; during this time, superfusion of saline was suspended. To wash 5-HT out after the treatment period, saline superfusion was reinstated at a high rate $(10-20 \mathrm{ml} / \mathrm{min})$ for 5 min, which was sufficient to completely exchange the solution in the recording chamber. Nerve activity was recorded throughout 5-HT exposure and during the first 5-10 min after washout was initiated. Each 5-HT application induced fictive calling, and fictive vocalizations could be induced multiple times in a single preparation; 5-HT application was repeated once per hour over the course of a day.

In seven male brains and four female brains, transection experiments were conducted to determine the anatomic components necessary for the production of fictive vocalizations. After one or two 5-HT applications (as above) established the baseline response to 5-HT, a series of scalpel cuts was made to remove portions of the brain or to sever a known point of fiber decussation. Thirty to $60 \mathrm{~min}$ after each cut, 5-HT application was repeated and nerve activity was recorded. Each condition was examined in four males and four females except when noted. The telencephalon and thalamic region were removed by making a cut in the coronal plane at the rostral extent of the optic tectum. In brains with the telencephalon removed, bilateral connections at the level of DTAM were severed by making a midsagittal cut through the tectum and cerebellum. In some cases, this bisection was a two-step process: tissue dorsal to the ventricle was cut first, and then ventral tissue was cut ( $n=3$ males). Finally, the region containing DTAM was removed with a coronal cut just rostral to nerve $\mathrm{V}$.

Electrical stimulation. Eight male brains were prepared for in vitro nerve recording as above (seven animals received an injection of HCG as above, one did not), but no 5-HT was applied to these brains. A tungsten electrode for delivering stimuli was placed at the presumptive location of DTAM: $\sim 475 \mu \mathrm{m}$ lateral of the midline at the border of the cerebellum and the optic tectum and descended slowly from dorsal to ventral. Electrical stimulus trains (square-wave voltage pulses, $50 \mu \mathrm{s}$ in length, frequency of $10-80 \mathrm{~Hz}$, variable amplitude) were generated using a Master-8 stimulator and delivered using an Iso-Flex stimulus isolation unit (A.M.P.I., Jerusalem, Israel) at various electrode depths. Motoneuron responses were evoked at depths of 100-400 $\mu \mathrm{m}$. At the end of the experiment, an electrolytic lesion was made to document electrode position ( $1.5 \mathrm{~mA}$ of direct current injection for $2 \mathrm{~s}$ ). Brains were fixed in $4 \%$ paraformaldehyde (PFA), sectioned at $60 \mu \mathrm{m}$, and stained with cresyl violet.

Analysis of vocal behavior. Advertisement call of male Xenopus has been described as having two main components: a slow trill, consisting of a series of clicks at $30-40 \mathrm{~Hz}$, and a fast trill, consisting of clicks at $50-70$ $\mathrm{Hz}$ (Kelley and Tobias, 1998). Here, we describe two additional subcomponents: "loud-slow" trills, which are high-amplitude, low-rate clicks immediately after the fast trill, and "transition clicks" of intermediate frequency, occurring between the slow and fast trills (see Fig. $1 \mathrm{~B}$ ). We 
defined a "bout" of calling as a single repetition of slow trill, transition clicks, fast trill, and loud-slow trill, in that order.

Ten song bouts were sampled randomly from each male, and instantaneous click rate was calculated as a reciprocal of interclick interval for each pair of clicks in a song bout using Raven (version 1.1; Cornell Lab of Ornithology, Ithaca, NY). Based on the instantaneous click rates, fast and slow trill segments within a calling bout were defined using the following decision rules. A frequency histogram of click rate for each animal showed a bimodal distribution and were well fit with two Gaussian curves $\left(R^{2}>0.9\right)$ with means and SDs of $\mu_{1}, \mu_{2}, \sigma_{1}$, and $\sigma_{2}$ (see Fig. $1 E$ ). A group of consecutive clicks with instantaneous frequency within $\mu_{1} \pm$ $2 \sigma_{1}$ was designated as slow trill, whereas a series of clicks with instantaneous frequency $>\mu_{2}-2 \sigma_{2}$ was designated fast trill (the two SD ranges never overlapped). We defined trills as continuous trains of similar click rate. Isolated clicks with a different frequency identity were occasionally found in the middle of a trill (i.e., a slow click in middle of fast trill or a fast click in middle of slow trill; $<5 \%$ of all clicks), and they were reclassified to match the surrounding trill. Clicks with instantaneous frequency that fell between the two SD ranges $\left(>\mu_{1}+2 \sigma_{1},<\mu_{2}-2 \sigma_{2}\right)$ were defined to be transitional clicks; such clicks always occurred after slow and before fast trill. Because there were very few transitional clicks per bout (zero to four), they were not analyzed as an independent trill type. However, these clicks were included in the count of total clicks per bout and the calculation of maximum sustained click rate (below). Rarely $(<1 \%)$, click rates would fall below the frequency range for slow trill $\left(<\mu_{1}-2 \sigma_{1}\right)$; these low click rates were caused by pauses in calling and were not included in analyses of click rate. Loud-slow trills do not differ substantially in click rate from slow trills; they were distinguished from slow trills in the sound waveforms based on their increased amplitude when compared with any other trill type.

Analysis of female vocalizations for animals used in transection experiments was similar: 10 bouts of release call were selected at random for each animal (a bout was defined as 5 or more clicks with click intervals $<1 \mathrm{~s}$ ), and instantaneous click rates were calculated as above. Female calls are monophasic, so no trill-type determination was necessary.

$\mathrm{T}$-female vocalizations were reviewed, and the production of different call types was documented. Because of the small number of vocal recordings obtained in this study, calls were not quantitatively analyzed; rather, data from a previous study of female and T-female vocalizations using identical recording and analysis procedures were used for comparison with in vitro recordings (Potter et al., 2005). Vocalizations recorded here were comparable with those reported previously.

Analysis of in vitro nerve recordings. Analysis of fictive advertisement call from male and $\mathrm{T}$-female brains paralleled the vocal analysis described above. Ten bouts of calling were selected at random for each animal (or, in the case of transection experiments, 10 bouts per treatment per ani$\mathrm{mal}$ ), and traces were rectified and low-pass filtered at $2 \mathrm{kHz}$ with Clampfit 9.2 software (Molecular Devices). CAPs corresponding to laryngeal motoneuron activity were identified in Clampfit using a threshold search (threshold set at $3 \sigma$ of background noise; minimum event duration, 0.4 $\mathrm{ms}$ ), and the start time and area of each CAP were measured. As with vocal analyses, instantaneous CAP rates were calculated. The frequency distribution of instantaneous CAP rates for fictive advertisement calling was bimodal, just as click rates were for in vivo advertisement call (see above). Therefore, identical processes were used to define slow and fast trill types, with one exception. Because sound amplitude information was not available for in vitro CAPs, loud-slow trills were defined by the following criteria (based on simultaneous nerve and vocal recordings in vivo): (1) instantaneous click rate in the "slow" range, (2) clicks immediately follow fast trill, (3) normalized CAP area $>25 \%$ of maximum.

Analysis was similar for recordings from female brains. Ten bouts of fictive release calling were selected at random from the recordings; CAPs were identified by threshold search, and instantaneous CAP frequencies and CAP area were measured.

Immunocytochemistry for tryptophan hydroxylase. To determine whether there are endogenous serotonergic projections to the vocal nuclei, we performed immunocytochemistry for tryptophan hydroxylase $(\mathrm{TrpOH})$. Nine males and five females were anesthetized with MS-222 and perfused transcardially with $4 \%$ PFA. Brain tissue sections were in- cubated with sheep anti-tryptophan hydroxylase primary antibody (Chemicon, Temecula, CA) at either 1:1000 (horizontal sections) or 1:50,000 (coronal sections) and $0.1 \%$ Triton X-100 in $0.1 \mathrm{M}$ phosphate buffer (PB) overnight at $4^{\circ} \mathrm{C}$. Sections were then incubated in biotinylated sheep IgG raised in goat (Chemicon) at 1:200 dilution with $0.1 \%$ Triton X-100 in PB, followed by incubation in ABC (Vector Laboratories, Burlingame, $\mathrm{CA}$ ) and staining with nickel-enhanced DAB solution (Vector Laboratories). No cells were labeled in control experiments when the primary antibody was omitted.

TrpOH-immunoreactive sections and adjacent Nissl-stained sections were imaged using an Olympus Optical (Melville, MY) microscope equipped with Magnafire acquisition software (Opelco via Olympus Optical). Eight-bit color images were taken at $10 \times$ and $20 \times$. Brightness and contrast of the entire image was optimized in Photoshop (Adobe Systems, San Jose, CA). Dorsal raphe neurons were identified by anatomic position (midline clusters of neurons just ventral to the ventricular system throughout the hindbrain) and TrpOH staining.

The premotor nucleus DTAM was retrogradely labeled from the motor nucleus IX-X (Brahic and Kelley, 2003; Zornik and Kelley, 2007), and its location was compared with TrpOH staining. Dextran-conjugated rhodamine crystals (molecular weight, 3000; Invitrogen, Carlsbad, CA) were deposited into the motor nucleus IX-X of five male brains using a minutin pin coated with dextran crystals, and the brains were incubated in oxygenated saline at $4^{\circ} \mathrm{C}$ for $96 \mathrm{~h}$. After fixing the tissue in $4 \% \mathrm{PFA}$ and sectioned in the coronal plane at $50 \mu \mathrm{m}$, neurons were visualized using a laser-scanning confocal microscope (Olympus Optical Fluoview). Twelve-bit $Z$-stack images were taken at 2.5 or $3 \mu \mathrm{m}$ increments using Olympus Optical Fluoview software (version 2.0) using the $10 \times$ objective and were then compiled and converted to eight-bit image files using NIH Image J.

Transneuronal label of raphe neurons. Transneuronal labeling using wheat germ agglutinin conjugated to horseradish peroxidase (WGAHRP) (Sigma) was used to determine whether the motoneurons in nucleus IX-X receive synaptic connections from the dorsal raphe nucleus. Four male frogs were anesthetized with MS-222 and transcardially perfused with $30 \mathrm{ml}$ of cold, oxygenated saline before their brains were isolated. Laryngeal motoneurons were retrogradely labeled with $10 \%$ WGA-HRP via laryngeal nerve. Brains were incubated in oxygenated saline at $9-10^{\circ} \mathrm{C}$ for $48 \mathrm{~h}$ to allow transneuronal labeling. The tissue was then fixed in 4\% PFA and sectioned in the horizontal plane at $50 \mu \mathrm{m}$. Tissue sections were reacted for HRP using tetramethylbenzidine stabilized with ammonium hepta-molybdate (Olucha et al., 1985). Tissue sections were then counterstained with cresyl violet. All four brains showed primary label of motoneurons with HRP, and two of four showed definitive secondary label in the raphe nucleus produced by transneuronal transport.

Statistical analyses. All statistical analyses were done using StatView software (SAS Institute, Cary, NC). For analyses of vocal and fictive advertisement call, the mean \pm SEM click (or CAP) rate for each trill type was calculated based on the final click type designations, as was the number of clicks of each trill type (fast and loud-slow) and the total number of clicks per bout. Because the number of slow clicks was variable and the frequency distribution skewed, the median was used to characterize the central tendency of this measure rather than the mean. The maximum sustained click rate, which has been used to evaluate Xenopus behavior previously (Potter et al., 2005), was calculated as follows: the average click rate for any 10 consecutive clicks gave the sustained click rate, and then the maximum of the sustained click rates was taken for each animal. CAP area was normalized to the percentage of maximum for each animal, and the mean CAP area for each trill type was calculated.

For analysis of female release call, we report the mean \pm SEM of instantaneous CAP rate. Maximum sustained CAP rate was calculated as above. Instantaneous and maximum sustained CAP rates for five females in this study was statistically compared with instantaneous and maximum sustained click rates for eight females published previously (Potter et al., 2005) using a Mann-Whitney $U$ test.

Both in vivo and fictive calls were obtained for 10 male animals, and the measures outlined above were compared for in vivo and fictive conditions using the Wilcoxon's signed-rank test. Multiple comparisons were 
corrected for by Bonferroni's correction: the $\alpha$ value was set at 0.0125 for each of the four click/CAP rate comparisons and for each of the four click/CAP number comparisons.

For transection experiments, advertisement calls were collected across a series of conditions in four male and four female brains: (1) in vivo vocalizations, (2) fictive, whole brain, (3) fictive, telencephalon and diencephalon removed, and (4) fictive, midbrain and DTAM bisected. Calls were analyzed as above (for males, six measures were analyzed for 10 bouts per condition per animal: rate of fast and slow trill, number of clicks or CAPs in fast and slow trill, and CAP size for fast and slow trill; for females, three measures were analyzed for 10 bouts per condition per animal: click or CAP rate, number of clicks or CAPs per bout, and CAP area). Each measure was analyzed across experimental conditions using repeated-measures $\mathrm{ANOVA}$, with condition as the repeated-measure variable. For any measure that yielded a statistically significant ANOVA, the Tukey-Kramer test was used as a post hoc test to determine differences between conditions.

A fifth transection condition (fictive, midbrain and DTAM removed) was also tested in all four of the female and one of the male brains above, as well as three additional male brains. Because no fictive calls were produced in condition 5 , no measures could be taken, and condition 5 was not considered in statistical analyses.

\section{Results \\ Application of 5-HT-induced fictive advertisement call in the isolated male brain}

Bath application of $30 \mu \mathrm{M} 5$-HT onto isolated male brains induced trains of CAPs in the laryngeal nerve that closely paralleled the timing and structure of the male Xenopus advertisement call as produced in vivo (Fig. $1 A, B$ ). The remarkable resemblance of the 5-HT-induced nerve activity to nerve recordings and call patterns from awake, vocalizing animals (described further in the analyses below) led us to conclude that 5-HT induced fictive advertisement call. Activation of fictive vocalizations in isolated brains indicates that there is a vocal CPG that can be activated by serotonin in the central vocal pathways.

Nerve recordings were obtained from the most caudal rootlet of nerve IX-X, which contains the axons of the laryngeal and glottal motoneurons, in adult male Xenopus laevis using an in vitro wholebrain preparation; thus, the central vocal pathway remained intact but isolated from sensory input and effector muscles. Recordings made before 5-HT application are mostly quiescent with spontaneous bursts of high-frequency, low-amplitude neural activity (observed once every few minutes, burst duration of $\sim 0.5 \mathrm{~s}$ ) (Fig. $1 C_{1}$ ) that correspond to glottal motoneuron activity; this fictive respiration closely resembles respiratory activity in vivo (Fig. $1 D_{1}$ ) and is associated with the opening of the glottis in a larynx-attached in vitro preparation (Zornik, 2006).

Within 10-70 s of 5-HT application (mean \pm SEM, $28.3 \pm$ $6.5 \mathrm{~s}$ ), activity consistent with fictive advertisement call was observed (Fig. 1A). The temporal pattern and shape of the CAPs strongly resembled the activity seen in laryngeal nerve recordings from awake, vocalizing animals (Fig. $1 B-D$ ) (Yamaguchi and Kelley, 2000). Each 5-HT application induced multiple bouts of advertisement call (mean \pm SEM, $61.3 \pm 27.8$; range, 6-300), often repeated one after another, although pauses in calling were also observed.

Because each CAP in the laryngeal nerve is faithfully translated into an audible click by larynx in vivo (Fig. $1 B$, inset) (Yamaguchi and Kelley, 2000), the trains of 5-HT-induced CAPs would have produced a series of clicks with an identical temporal profile had the nerve been connected to the larynx. Thus, the temporal pattern of CAPs recorded from the laryngeal nerve in vitro can be quantified and directly compared with the audio recordings made from the awake, vocalizing frog from which the brain was isolated.
We found that fictive advertisement calls showed very similar temporal structure to vocalizations recorded previously from the same animals. Fictive calls showed the same stereotyped call structure as seen in vivo, with all of the components of the advertisement call (slow trill, transition, fast trill, and loud-slow trill) clearly identifiable and in the correct sequence (Fig. $1 B, F$ ). Statistical comparison revealed a slight reduction in CAP rates for fictive vocalizations when compared with click rates for in vivo calls. Mean instantaneous CAP rates for slow, fast, and loud-slow trills were significantly slower for fictive vocalizations than for in vivo vocalizations (Wilcoxon's signed-rank test; $z=-2.701$, -2.803 , and -2.547 , respectively; $p<0.0125$ for all) (Fig. $2 A$ ). The maximum sustained frequency of fictive vocalizations was also significantly slower than in vivo vocalizations (Wilcoxon's signed-rank test; $z=-2.803 ; p=0.005$ ) (Fig. $2 A$ ). Reductions in the frequency of rhythmic output are common in CPGs that have been isolated from sensory feedback (Wilson, 1961), and the reductions in frequency observed here (typically 10-20\%) were relatively minor.

Despite the slowing of the advertisement call rhythm during fictive calling, the relationship between slow and fast trill rates was constant across fictive and in vivo calls. The absolute difference between fast and slow trill rates was $24.95 \pm 3.18 \mathrm{~Hz}$ in vivo and $24.94 \pm 4.02 \mathrm{~Hz}$ for fictive calling (Wilcoxon's signed-rank test; $z=-0.153 ; p=0.88$ ), suggesting that the mechanism that switches between slow and fast trill rhythms operates at a constant frequency range both in vivo and in vitro.

The number of clicks or CAPs contained in each bout of song was similar for in vivo and fictive advertisement call (43.7 \pm 12.6 clicks/bout in vivo, 42.2 $\pm 16.3 \mathrm{CAPs} /$ bout fictive; Wilcoxon's signed-rank test; $z=-0.764 ; p=0.44$ ) (Fig. $2 B$ ). However, the distribution of these clicks into the different trill types was altered somewhat (Fig. $2 B$ ). Fictive fast trills contained more clicks, on average, than in vivo calls $(z=-2.701 ; p=0.007)$, whereas fictive loud-slow trills $(z=-2.395 ; p=0.017 ; \alpha=0.0125)$ and slow trills $(z=-1.070 ; p=0.28)$ were not significantly different. Thus, bout length and structure, as measured by the number of clicks, remains predominantly the same in the fictive preparation, implying that the mechanisms responsible for transitioning from one portion of the song to the next remain essentially intact in the isolated brain.

Although fictive advertisement call and respiration were the predominant forms of activity observed during in vitro nerve recordings, patterns of activity associated with other male vocalizations were also observed. Long bouts consisting of only slow trill have been observed in behaving males, interspersed with advertisement call; likewise, long series of CAPs, consistent in shape and frequency with slow trill, were observed occasionally after serotonin application in vitro (Fig. $3 A$ ). Fast trill was never observed alone but was always preceded by slow trill both in vivo and in vitro, suggesting that the generation of fast trill requires production of slow trill but not vice versa. The male call repertoire also includes other call types, including amplectant call and release call (male release call, indistinguishable from female release call, has also been called "male ticking") (Kelley and Tobias, 1998; Tobias et al., 2004). Fictive amplectant-like calls were observed in 4 of 10 brains (Fig. 3B) and have been observed previously to occur spontaneously in in vitro preparations (Zornik, 2006). Bouts of fictive male release call were also observed in 3 of 10 male brains in response to 5-HT application (Fig. 3C). In vivo, release call is common in juvenile males but rare in adults (Oberlander et al., 2004; Tobias et al., 2004). No fictive calls were ob- 

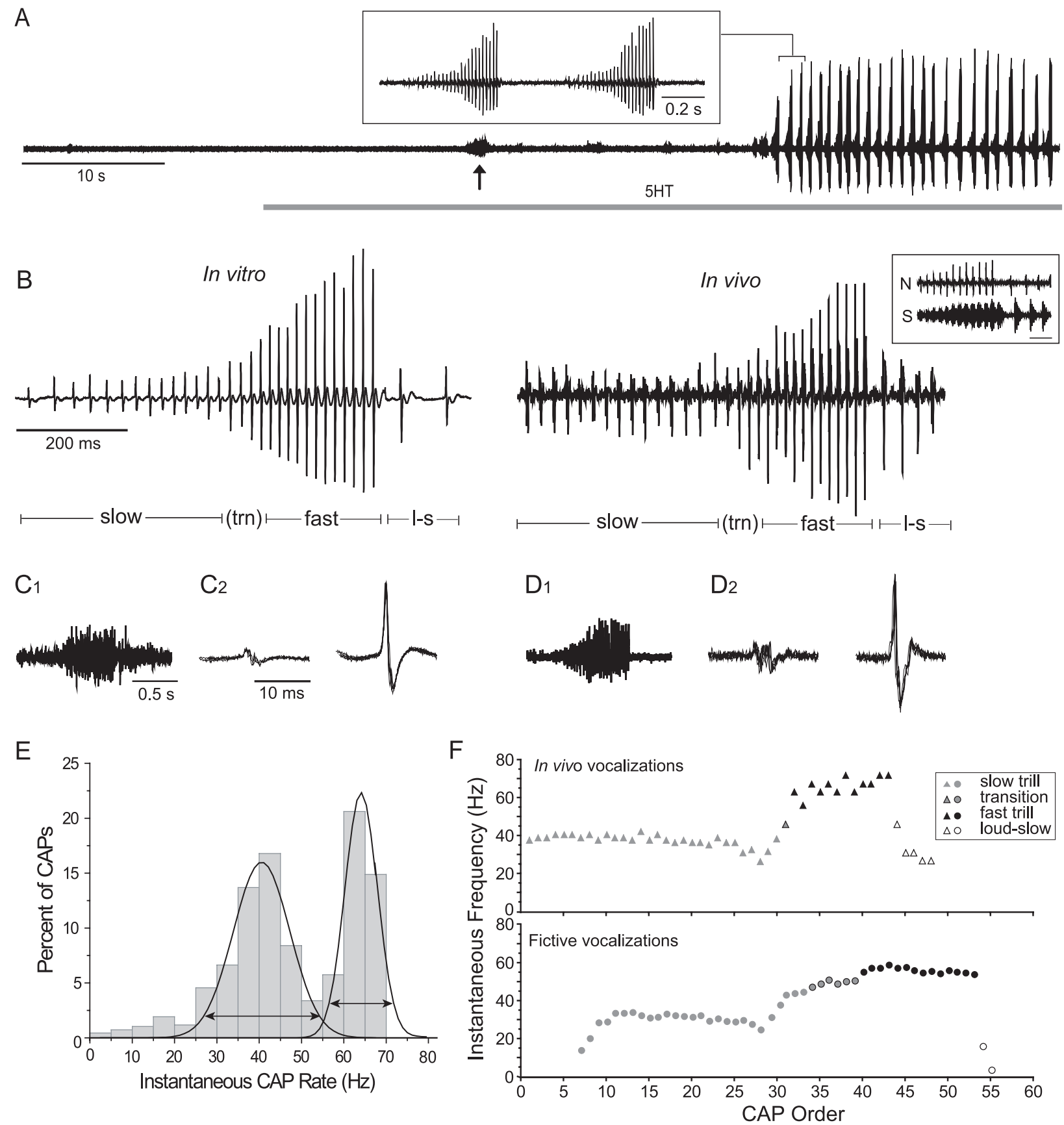

Figure 1. Male fictive vocal patterns resemble nerve recordings of in vivo advertisement call. $A$, In vitro nerve recording before and after 5 -HT application shows the onset of fictive advertisement call $\sim 35$ s after 5 -HT was added to the bath (gray bar). The only other activity apparent in the recording is respiratory activity (arrow). $\boldsymbol{A}$, Inset shows bracketed region in $\boldsymbol{A}$ with an enlarged timescale, demonstrating that each of the large-amplitude bursts on the right is a bout of fictive advertisement call. B, Fictive (left) and in vivo (right) nerve recordings of male advertisement call showing slow, fast, and loud-slow (I-s) trills, as well as transition (trn) CAPs between slow and fast trills. $\boldsymbol{B}$, Inset, Simultaneous recordings of nerve activity and sound production in a freely behaving frog. Traces show a portion of fast and loud-slow trill; each CAP in the nerve recording ( $\mathrm{N}$; top) is followed by the production of a click of sound (S; bottom trace) several milliseconds later. $S$ cale bar, 50 ms. $C$, $\boldsymbol{D}$, Fictive $\left(\boldsymbol{C}_{1}\right)$ and in vivo $\left(\boldsymbol{D}_{1}\right)$ nerve recordings of glottal bursts associated with respiration; enlarged traces of slow (left) and fast (right) trill CAPs for fictive $\left(\boldsymbol{C}_{2}\right)$ and in vivo $\left(\boldsymbol{D}_{2}\right)$ recordings in $\boldsymbol{B}_{\text {; five }}$ traces are overlaid for each. Timescales are the same for $\boldsymbol{C}_{1}$ and $\boldsymbol{D}_{1}$, as well as for $\boldsymbol{C}_{2}$ and $\boldsymbol{D}_{2}$. $\boldsymbol{E}$, Frequency histogram showing the bimodal distribution of instantaneous $C A P$ rates for one individual fit with two Gaussian curves. CAPs within 2 SD of the peak of the lower distribution were designated as slow trill, and those within 2 SD of the upper distribution were fast trill (ranges marked with arrows). Values falling between those ranges were transitional clicks; for additional detail on CAP designations, see Materials and Methods. $F$, Instantaneous CAP frequency versus CAP order for one bout of in vivo (top) and one bout of fictive (bottom) advertisement call, showing CAP designation as slow, transition, fast, or loud-slow trill.

served resembling other male calls, such as growling or chirping (Tobias et al., 2004).

In isolated female brains, fictive release call can be induced by 5 -HT

5-HT also activated the central vocal pathways in the isolated female brain, inducing fictive release calling (Fig. 4A). 5-HT- induced trains of CAPs resembled female release calls recorded in vivo; there was no significant difference in instantaneous or maximum sustained rates when compared with a previously published description of in vivo release call (Mann-Whitney $U$ test; instantaneous rate, $\mathrm{z}=0, p>0.99$; maximum sustained rate, $z=$ $-0.945, p=0.345$ ) (Fig. 4B) (Potter et al., 2005). Thus, unlike advertisement call, fictive release call did not show a reduction in 

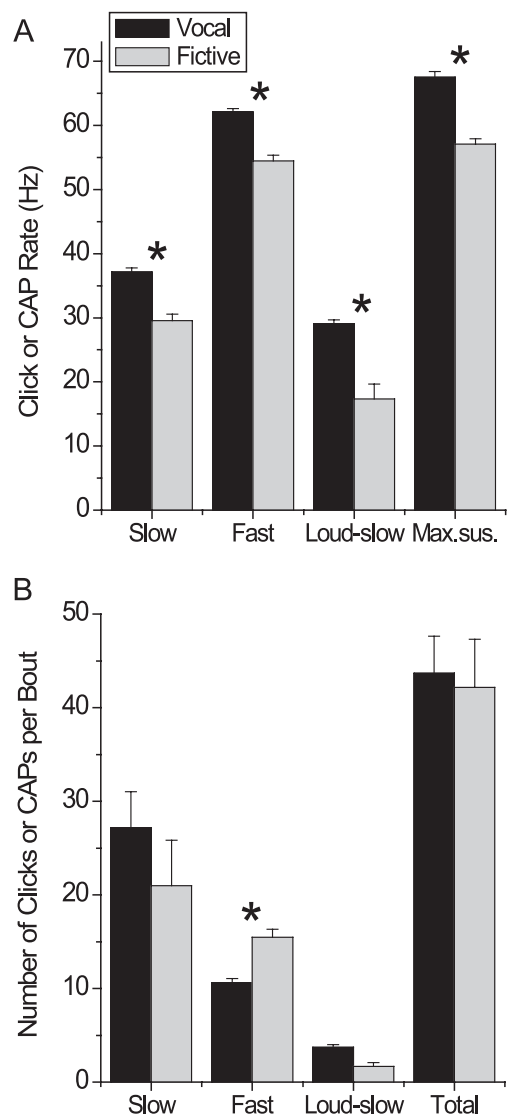

Figure 2. A comparison of fictive and in vivo male advertisement call. $A$, Instantaneous CAP rates for slow, fast, and loud-slow trills, as well as the maximum sustained CAP rate, for fictive advertisement call are lower than click rates produced by the same animals in vivo. B, Number of clicks and CAPs per bout for each trill type are compared for the in vivo and in vitro conditions. Total includes slow, fast, loud-slow, and transition. $n=10$ animals for each bar; error bars are SEM. Compared with Wilcoxon's signed-rank test; ${ }^{*} p<0.0125$.

rate relative to in vivo behavior. The size and shape of the CAPs closely resembled published descriptions of nerve activity during female release calling (Yamaguchi and Kelley, 2000). There were no apparent differences in fictive calling between gonadectomized $(n=2)$ and intact $(n=6)$ females.

Five of eight female brains required more than one (between two and four) $10 \mathrm{~min}$ application of 5-HT (1 h apart) before fictive release calls were observed. During trials in which fictive release calls were produced, the latency of the first fictive vocalizations was much longer than in male brains (mean \pm SEM, $161.1 \pm 47.3 \mathrm{~s}$; nearly six times longer than male latency). Once evoked, fictive release calling outlasted the serotonin application, typically continuing for several minutes after 5-HT washout. Thus, bath-applied 5-HT appeared to have a different time course in male and female brains in vitro; the male vocal system responded rapidly to the introduction and withdrawal of 5-HT, whereas the female vocal system required greater 5-HT exposure to induce fictive behavior, which then persisted after washout.

\section{5-HT induced male- and female-like vocalizations in testosterone-treated females}

Female Xenopus remain receptive to androgen in adulthood. Chronically administering testosterone to ovariectomized females masculinizes vocal behavior within 2-3 months and has been shown to alter the morphology and function of neurons in the vocal pathway (Hannigan and Kelley, 1986; Yamaguchi et al.,

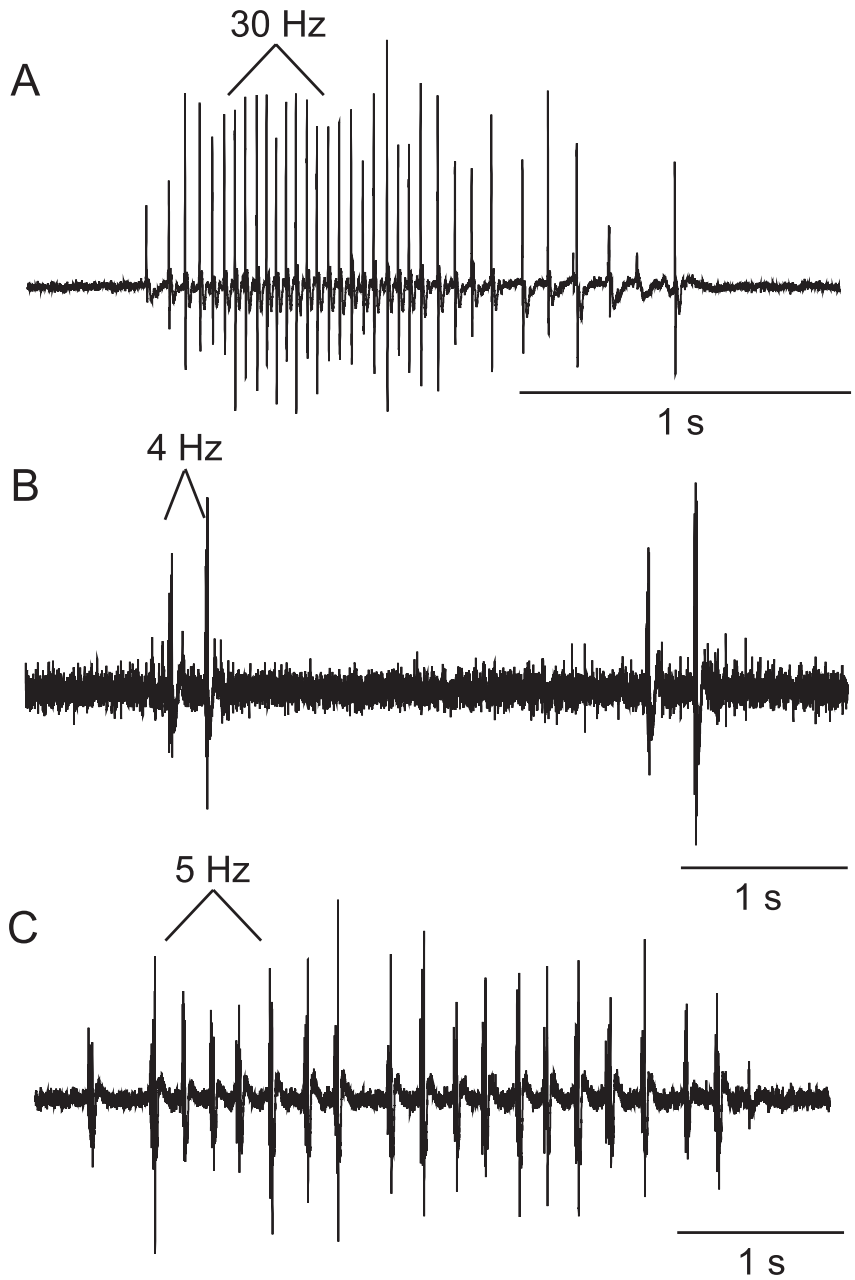

Figure 3. Several male calls were produced as fictive vocalizations. $\boldsymbol{A}$, An example of fictive slow trill without fast trill. $\boldsymbol{B}$, An example of fictive amplectant call. $\boldsymbol{C}$, An example of fictive male release call. Average CAP rates are given for a short series of CAPs from each trace.

2004; Potter et al., 2005). To determine what effects testosterone had on the vocal CPG, we examined the 5-HT-induced fictive calls produced by four $\mathrm{T}$-female brains in vitro. We compared the temporal structure of T-female fictive vocalizations with those of males and untreated females as well as in vivo vocalizations from T-females.

Three of four T-female brains (one with 5 months and two with 8 weeks testosterone exposure) produced fictive vocal activity consistent with the male advertisement call (i.e., clearly delineated, alternating fast and slow trills) (Fig. 5A). The maximum sustained CAP rate as well as the CAP rates for slow and fast trills were comparable with those seen for T-female vocalizations in vivo, and, for one T-female brain (the brain with the longest testosterone exposure), these measures fell within the range of male fictive vocalizations (Fig. 5B). Additionally, the latency for 5 -HT induction of fictive vocalizations was $10-20 \mathrm{~s}$, similar to that observed in males, indicating that the mechanism by which 5 -HT activated vocal patterns in vitro was also masculinized in these animals.

We obtained in vivo sound recordings of advertisement calllike vocalizations for two of the $\mathrm{T}$-females that produced fictive advertisement call (Fig. 5A); both had 8 weeks testosterone exposure (no vocal recordings were obtained for the 5 month exposure animal). Interestingly, in both cases, the fictive call was a 
A

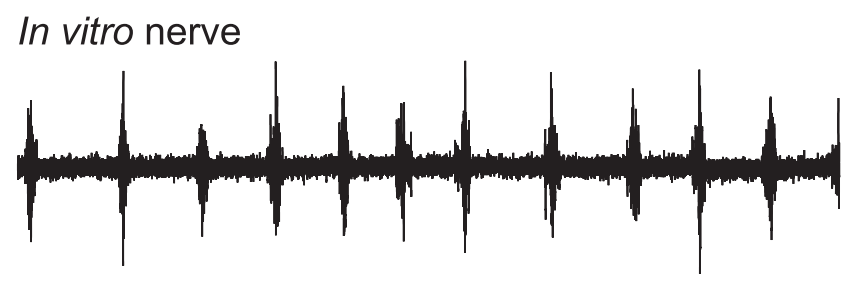

In vivo sound
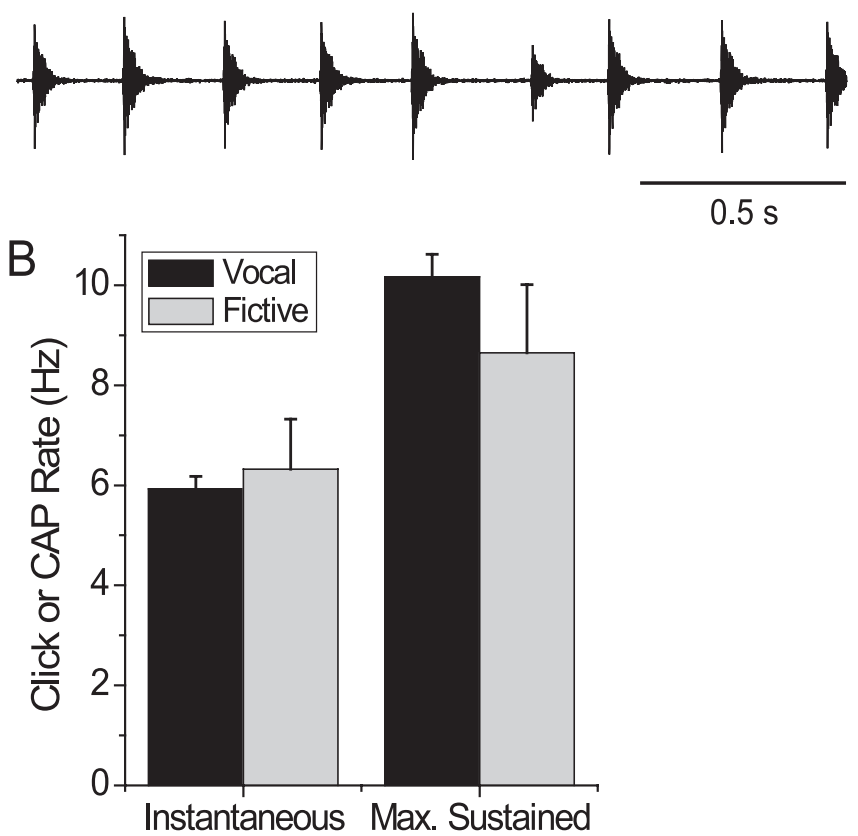

Figure 4. Female brains produce sex-specific fictive vocalizations. $\boldsymbol{A}$, Fictive release call (top) compared with an audio recording of a release call in vivo (bottom). $\boldsymbol{B}$, CAP rates for female fictive calls are similar to those observed in vivo. Vocal data are from Potter et al. (2005). Bar graph shows means, and error bars are SEM.

better facsimile of male call structure than was the sound produced in vivo. For instance, in Figure 5, the fast trills of the in vivo vocalization show instantaneous click rates of $55 \mathrm{~Hz}$ but are only two or three clicks long and separated from the slow trill by pauses, as if a portion of each bout is not being executed, features not seen in male calls. The fictive call produced by the brain of the same animal, however, is qualitatively indistinguishable from male fictive call, with a normal-length fast trill and smooth transitions between different trill types. This suggests that the brain may be more plastic than the periphery, at least in adulthood, showing more complete masculinization in response to testosterone.

Female-typical fictive release calling was also observed in three T-female brains (all with 8 weeks testosterone exposure). These calls were indistinguishable from those produced by untreated female brains, as described above (instantaneous CAP rate, $5.6 \pm$ $3.1 \mathrm{~Hz}$ ). Two of these animals were also observed to produce release call vocalizations in vivo when paired with a male. This demonstrates that testosterone exposure, at least at 8 weeks, does not impact the ability to produce female-typical call patterns.

Testosterone exposure, therefore, induces the formation of a masculine vocal CPG, altering both the temporal pattern of motoneuron output and the time course in which 5-HT can activate vocal production. These added functions do not exclude the production of the sexually undifferentiated release call, a finding also supported by the observations of fictive release call in male brains (above).

\section{Progressive transection reveals that DTAM region is necessary}

Having determined that Xenopus vocal production is governed by one or more sexually dimorphic CPGs and that pattern generation can be activated by 5 -HT in both sexes, we conducted a series of experiments to determine which elements of the known vocal circuitry might be responsible for 5-HT-induced pattern generation. We severed the vocal pathway at three points in male and female brains to determine which vocal nuclei were necessary for production of 5-HT-induced fictive vocalizations in each sex.

Removal of the telencephalon and thalamic region (e.g., transection at the level of the rostral extent of the optic tectum) did not inhibit the production of fictive calls in either males or females (Fig. $6 A_{1}-A_{3}$ ). Female call structure was unaffected. However, the structure of the male advertisement call was modified slightly after the removal of telencephalon; the length of the fast trill was significantly elongated (93\% increase; $16.5 \pm 1.4$ CAPs per bout before vs $31.7 \pm 4.9$ CAPs per bout after transection, mean \pm SEM; Tukey-Kramer test, $p<0.05$ ) (Fig. $6 B$ ). This was not attributable to acute trauma, because the effect was still present up to $12 \mathrm{~h}$ after transection. Thus, the telencephalon and thalamic region are neither critical components of the vocal CPG nor the site of action for 5-HT but may limit the length of the fast trill.

Bisection of the region containing the premotor nucleus DTAM, which cut projections from DTAM to the contralateral motor nucleus, as well as any decussating fibers between the two DTAM nuclei (Zornik and Kelley, 2007), did not inhibit 5-HTinduced fictive calls in either sex (Fig. 6 $A_{4}$ ) or alter female call structure (Fig. $6 B$ ). The effects of the bisection on call parameters were further examined in males. Decussating fibers from DTAM pass ventral to the fourth ventricle (Zornik and Kelley, 2007); predictably, when bilateral connections dorsal to the ventricle were bisected, no change was observed in the structure of fictive advertisement call (data not shown). However, when bilateral connections ventral to the ventricle were bisected, fast trill became significantly degraded, whereas slow trill remained intact (Fig. 6 B). Fast trill CAPs showed a $72 \%$ reduction in size $(70.1 \pm$ $3.4 \%$ normalized CAP area for the whole brain vs $19.9 \pm 3.7 \%$ after bisection; Tukey-Kramer test, $p<0.05$ ), as well as an $8.2 \%$ reduction in rate (Tukey-Kramer test, $p<0.05$ ). The results suggest that bilateral connections in the ventral brainstem at the level of DTAM help shape fast trill but not slow trill or release call. Interestingly, the length of the fast trill, which was elongated by telencephalon removal, recovered after the bisection.

When the region containing DTAM was removed bilaterally by cutting the hindbrain just caudal to the cerebellum, no fictive advertisement or release calling, or any recognizable vocal activity, was observed for either sex (Fig. 6 $A_{5}$ ). As many as five applications of 5 -HT over a $7 \mathrm{~h}$ period failed to elicit fictive activity. Normal respiratory activity (glottal bursting) was observed after the removal of DTAM, as well as an increase in asynchronous and arrhythmic background activity during the 1-2 min after 5-HT application. This noise response may be attributable to unpatterned excitation of laryngeal motoneurons, glottal motoneurons, or both. This result indicates that the region containing DTAM is necessary to induce fictive vocal patterns as a critical part of the vocal CPG, as a critical site of action of 5-HT that then initiates vocal activity, or both. The similarity of the results in male and female brains suggests that the basic structure of the 

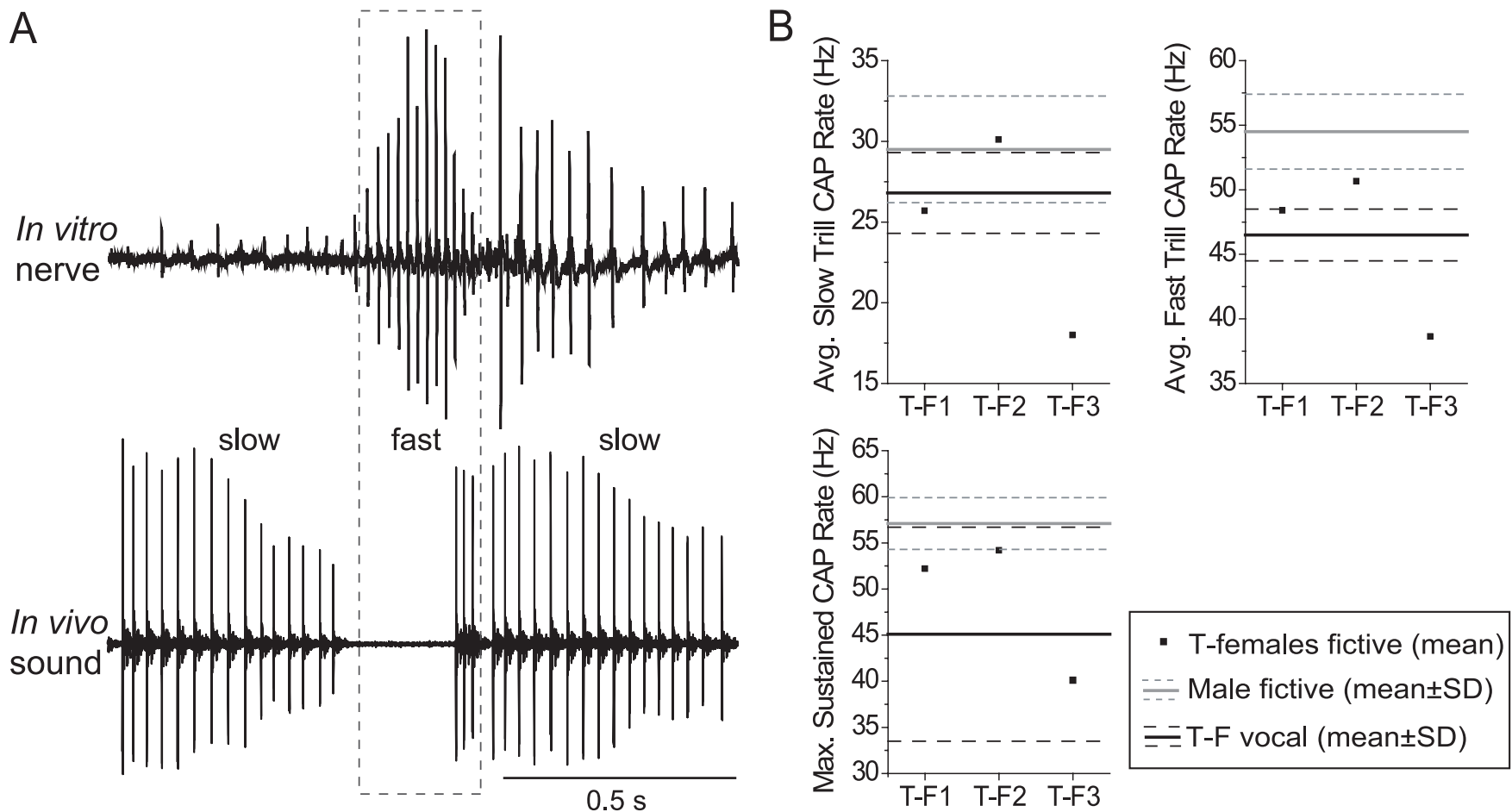

Figure 5. T-females produce male-like vocal patterns in vivo and in vitro. A, Fictive (top) and vocal (bottom) recordings are from one animal after 8 weeks of testosterone exposure. Slow and fast trill segments are marked. The fictive call resembles male advertisement call more closely than the sound produced in vivo, which shows an abnormal pause between slow and fast trill. $\boldsymbol{B}$, Fictive advertisement calls produced by three T-females are compared with fictive calls from male brains and in vivo calls by T-females. Dots are means CAP rates for the three T-females that produced advertisement call in this study. Gray and black solid lines are means for male fictive and T-female vocalizations, respectively; dashed lines are \pm 1 SD. T-female vocal data from Potter et al. (2005); male data are the same as shown previously (Fig. 2).

vocal circuit and possibly even the location of the pattern generator are shared among the sexes.

\section{Electrical stimulation of DTAM is sufficient to drive fictive calling}

With evidence for a critical role for DTAM in the production of 5-HT-induced fictive vocal behavior from the transection experiments, we tested the ability of DTAM to evoke fictive advertisement call in the absence of exogenous 5-HT by applying local electrical stimulation to DTAM. Of eight male brains tested, two produced what appeared to be fictive advertisement call in response to electrical stimulation delivered at $20-40 \mathrm{~Hz}$ (Fig. 7A). Activity was considered to be fictive calling if it met the following criteria: CAPs occurred in bouts resembling advertisement call structure (slow trill followed by fast trill), CAP shapes resembled CAPs produced during in vivo advertisement call (Fig. 1), CAPs did not show a single, fixed latency to the stimuli, nor was there exclusively a 1:1 relationship of stimuli to CAPs (stimuli may be followed by zero or more than one CAP). For both brains, bouts of fictive activity occurred at variable latency from the onset of stimulation; there were periods of several seconds at the beginning of each trial as well as times in the middle of trials when stimuli would produce no response (Fig. 7A).

The fictive activity evoked by electrical stimulation was remarkably similar to that evoked by 5 -HT. The maximum sustained frequencies for fictive activity in the two electrical stimulation brains were 53.2 and $56.3 \mathrm{~Hz}$. Fast trill length and rate were also similar to 5-HT-induced fictive calling and were independent of stimulus frequency, at $12.9 \pm 10.3$ CAPs per bout and $49.0 \pm 6.5 \mathrm{~Hz}$ on average, respectively (mean $\pm \mathrm{SD}$ ). Fast trill rate did not vary systematically with stimulus frequency (Fig. 7D).
Furthermore, the distribution of CAP latencies from the preceding stimulus was random (Fig. $7 E$ ), showing that the fictive fast trill was a free-running rhythm, independent of the stimulus rhythm, and was not simply a result of each stimulus driving multiple CAPs.

Slow trill length was variable, as with 5-HT-induced fictive calls, ranging from 4 to 28 CAPs per bout. Slow trill rate appeared to depend, in part, on the rate of stimulation: $20 \mathrm{~Hz}$ stimulation produced slow trill near $20 \mathrm{~Hz}, 30 \mathrm{~Hz}$ stimulation led to slow trill just shy of $30 \mathrm{~Hz}$, and $40 \mathrm{~Hz}$ stimulation led to still faster slow trill, although it did not reach $40 \mathrm{~Hz}$ (Fig. 7D). Furthermore, the distribution of latencies for slow trill CAPs was not uniform (Fig. $7 E$ ), indicating entrainment by the stimulus; this entrainment was most powerful at $20 \mathrm{~Hz}$ stimulation and lessened with each increase in stimulus rate.

The entrainment of the slow trill rhythm by the stimulus suggests that DTAM stimulation was directly affecting, or possibly even serving as, a slow trill pacemaker. Only stimulus frequencies of 20,30 , or $40 \mathrm{~Hz}$ (rates consistent with slow trill) were effective at generating fictive activity patterns, and these stimulus frequencies evoked fictive calling in most trials; at stimulus frequencies of $10,50,60$, and $80 \mathrm{~Hz}$, either small, 1:1 responses or no responses were recorded. It is interesting that DTAM stimulation seemed to influence the temporal pattern of slow trill but not fast trill, suggesting that rhythmogenesis for fast and slow trills may be separable processes.

In six additional brains, DTAM stimulation resulted only in small CAPS in the laryngeal nerve, which had a fixed 1:1 relationship with stimuli at a set latency of $<10 \mathrm{~ms}$ (data not shown), similar to previous reports (Rhodes and Yamaguchi, 2005; Zornik, 2006). Histological examination revealed that the stimu- 

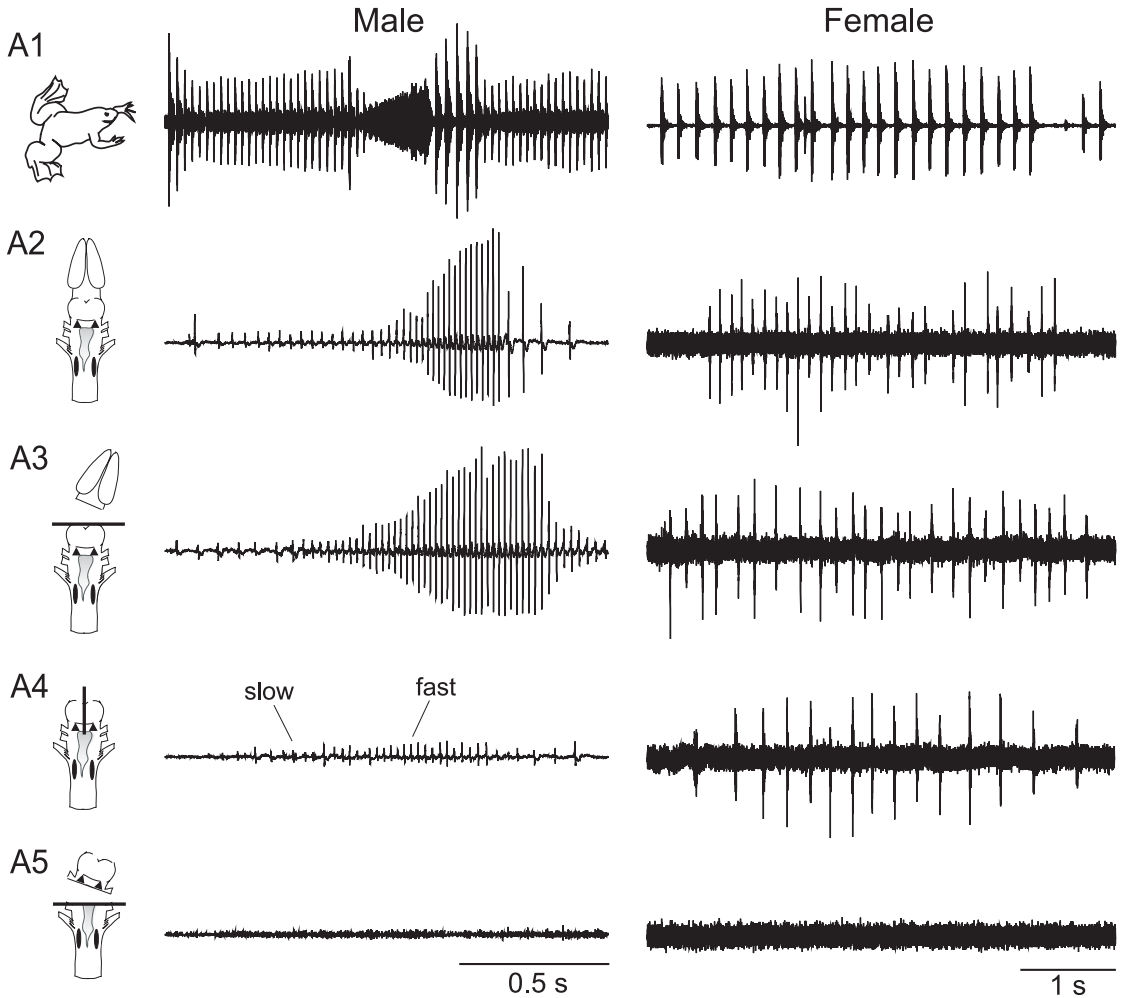

B
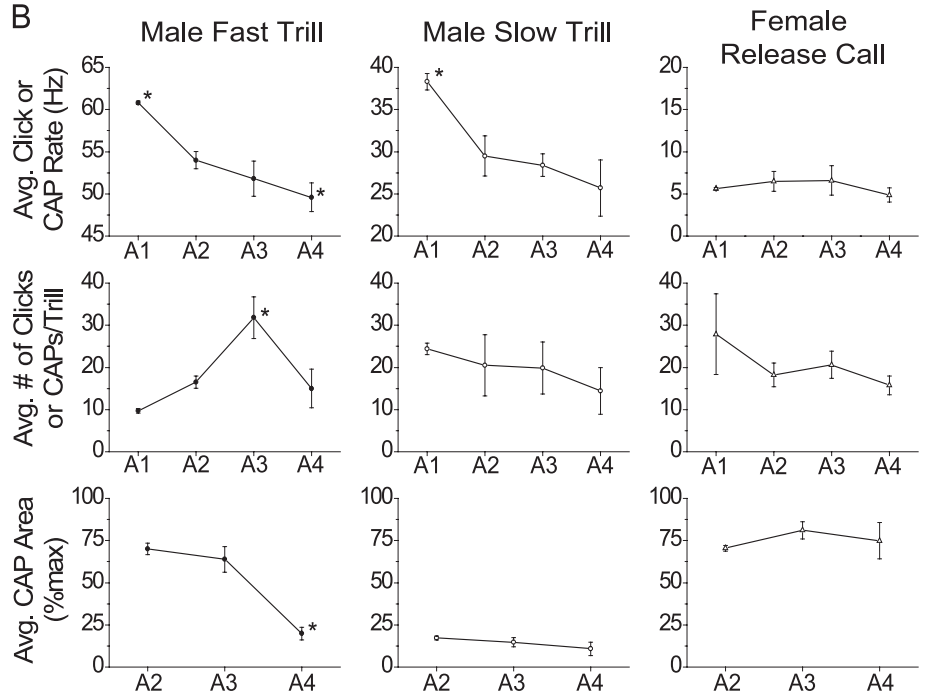

Figure 6. Transection experiments show that DTAM is necessary for 5-HT-induced fictive vocalizations in both sexes. $\boldsymbol{A}$, Examples of male advertisement call (middle column) and female release call (right column) in vivo, in vitro, and after a series of transections. Drawings (left column) show the condition of the brain during each recording. The location of DTAM is marked with black triangles; nucleus IX-X is marked with black ovals. All traces in each column are from a single animal, except the in vivo female recording. $\boldsymbol{A} \mathbf{1}$, Sound recordings in vivo. $\boldsymbol{A} \mathbf{2}$, Nerve recordings of fictive calls in the whole brain. $\boldsymbol{A} \mathbf{3}$, Fictive call after the telencephalon and diencephalon were removed revealed lengthened fast trill for males and normal call for females. A4, Fictive call after bisection of the midbrain and rostral brainstem resulted in reduced fast trill amplitude and rate in the male (slow and fast trill segments are marked) but no change in the female call. $A 5$, Removal of the rostral brainstem (containing DTAM) resulted in the loss of all fictive vocalizations in both males and females. $\boldsymbol{B}$, Plots showing CAP rate (top row), number of CAPs per bout (middle row), and CAP area (bottom row) for male fast trill (left column), male slow trill (middle column), and female release call (right column) for the conditions illustrated in A1-A4. $n=4$ for all. Analyzed with repeated-measures ANOVA followed by TukeyKramer post hoc test. ${ }^{*} p<0.05$ on Tukey-Kramer comparing marked condition with whole-brain fictive condition (A2).

lating electrode was positioned at the dorsorostral edge of the DTAM nucleus in all eight brains. Thus, stimulation may have been acting on DTAM somata or possibly on axons entering DTAM. This location is not consistent with axons exiting DTAM (Zornik and Kelley, 2007).
Electrical stimulation of DTAM, like the transection experiments above, suggests that it is a critical component of the vocal pattern-generating circuitry. DTAM is necessary for the production of 5-HTinduced fictive calling, and electrical stimulation of DTAM is sufficient to produce fictive vocalizations without exogenous 5-HT.

\section{Serotonergic projections to the central vocal pathways}

The experiments above revealed that exogenous 5-HT acts on vocal circuits in the brainstem to evoke fictive vocalizations. To determine whether there are endogenous serotonergic projections to vocal nuclei in the brainstem (including DTAM, the inferior reticular formation, and motor nucleus IX-X), we performed immunocytochemistry for the rate-limiting enzyme in serotonin synthesis, $\mathrm{TrpOH}$, in male and female Xenopus brains.

Specific cytosolic staining of TrpOHpositive cell bodies was observed in the raphe nuclei, and $\mathrm{TrpOH}$-positive axons originating from raphe neurons were seen projecting to other brainstem nuclei. No sex differences were observed in raphe neuron staining or in the TrpOH-positive projection pattern described below.

At the rostrocaudal level of motor nucleus IX-X, labeled axons could be traced from the raphe cells laterally through the reticular formation and nucleus IX-X (Fig. 8A,B). The TrpOH-positive axons appeared to have numerous varicosities, consistent with possible 5-HT release into these nuclei. To test whether these projections are likely to represent synaptic contacts, a transneuronal tracer was used. After motoneurons were backfilled with WGA-HRP, secondary label could be detected in dorsal raphe neurons (Fig. $8 C, D)$, providing additional evidence that serotonergic neurons of the dorsal raphe could directly provide endogenous 5 -HT to laryngeal motoneuron.

In the rostral hindbrain at the level of DTAM, TrpOH-positive terminal fields were seen throughout DTAM and the area immediately surrounding the nucleus (Fig. 7E). Anatomical landmarks were confirmed by comparison of $\mathrm{TrpOH}-$ labeled tissue to adjacent sections that were stained for Nissl substance (Fig. 7F) as well as comparison with tissue sections from other brains in which DTAM cells were retrogradely labeled from nucleus IX-X using rhodamine (Fig. 7E, inset).

Thus, serotonergic neurons from the dorsal raphe nucleus project to premotor nuclei, including DTAM and possibly the reticular formation, as well as to the motor nucleus, in both sexes, 
suggesting that endogenous release of serotonin to these nuclei may trigger or facilitate vocalizations in vivo.

\section{Discussion}

We demonstrated that isolated Xenopus brains can generate sex-specific fictive vocal patterns. Sensory feedback is not necessary to generate or maintain rhythms; rather, vocal circuitry in the CNS is sufficient for creating precisely timed, patterned vocal output. Thus, we conclude that these Xenopus vocalizations derive from a sexually dimorphic CPG.

Vocal activity patterns in Xenopus are remarkably well preserved in the in vitro preparation. In other systems, such as the rodent spinal cord, isolating the locomotor CPG from sensory input results in rhythms that are slower by an order of magnitude (Cowley and Schmidt, 1994). In Xenopus, conversely, a $10-20 \%$ decrease in rate was observed for male advertisement call, and no reduction was observed for female release call. The difference between these systems may lie in the degree to which the CPG is modulated by sensory feedback. Unlike muscles involved in locomotion, laryngeal muscles do not bear a changing load and contain no known proprioceptors to provide sensory feedback (Sassoon et al., 1986). Other sources of sensory feedback (i.e., auditory) may modulate vocal patterns in vivo, but it seems that feedback plays a relatively small role in maintaining vocal rhythms.

\section{A hindbrain CPG}

Based on our transection experiments, we propose that the area including the optic tectum and the rostral brainstem contains a critical element for vocal production. Removal of more rostral structures did not disrupt fictive vocal production, but removal of this region abolished 5-HTinduced vocal activity, indicating that it contains a critical portion of the vocal CPG, the site of action for 5-HT, or both. Electrical stimulation of this region was sufficient to activate the CPG and induce fictive vocalizations, strengthening the evidence that this region is important for pattern generation. Finally, when commissural connections in this region were severed in males, fast trill CAP amplitude was reduced drastically and CAP rate slowed, suggesting that decussating axons are necessary for proper fast trill execution.

The most notable vocal structure in this region is the premotor nucleus DTAM, which is robustly and reciprocally connected with nucleus IX-X (Wetzel et al., 1985; Brahic and Kelley, 2003; Zornik and Kelley, 2007) and receives serotonergic projections from the dorsal raphe nucleus (above). Thus, DTAM seems to be a critical component of the 5-HT-sensitive CPG circuit. DTAM is located in a region of the brain frequently associated with rhyth-
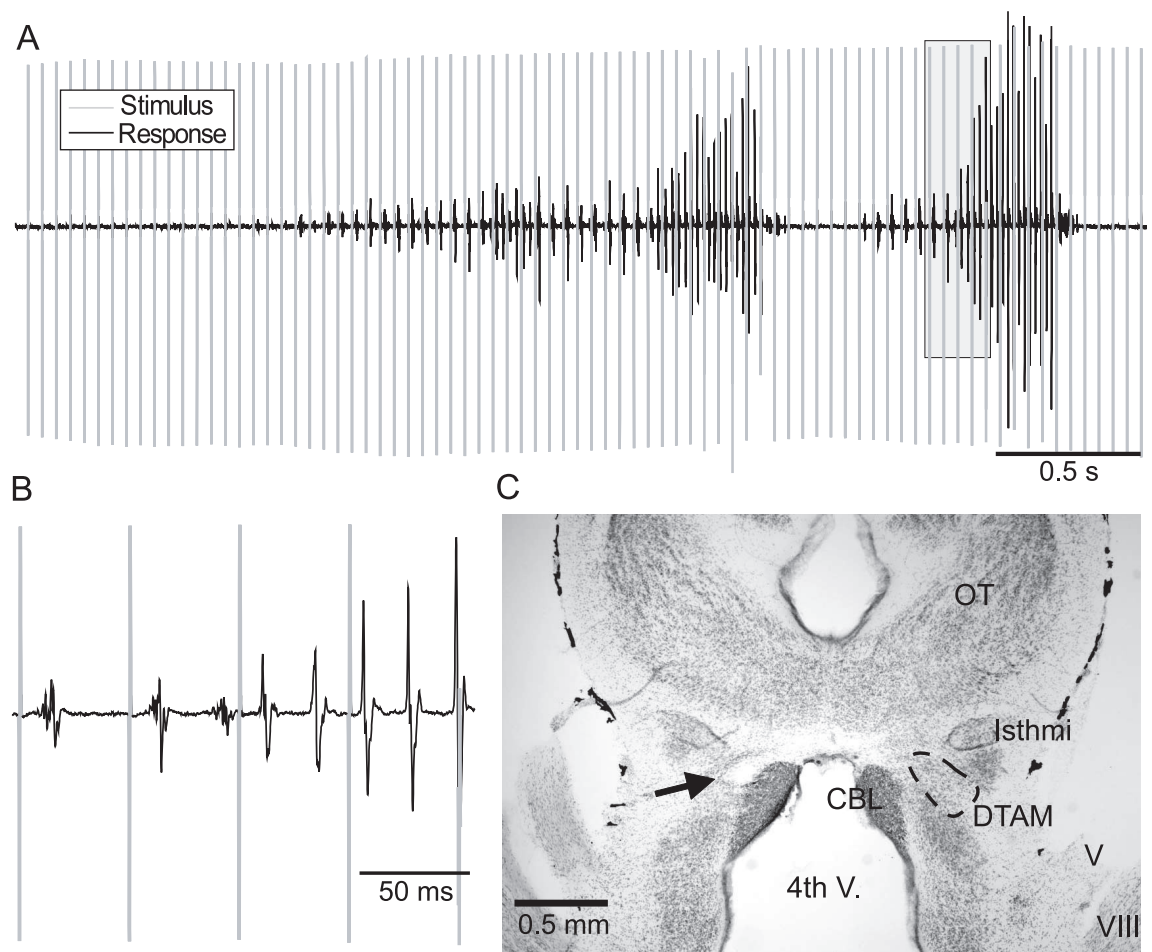

c
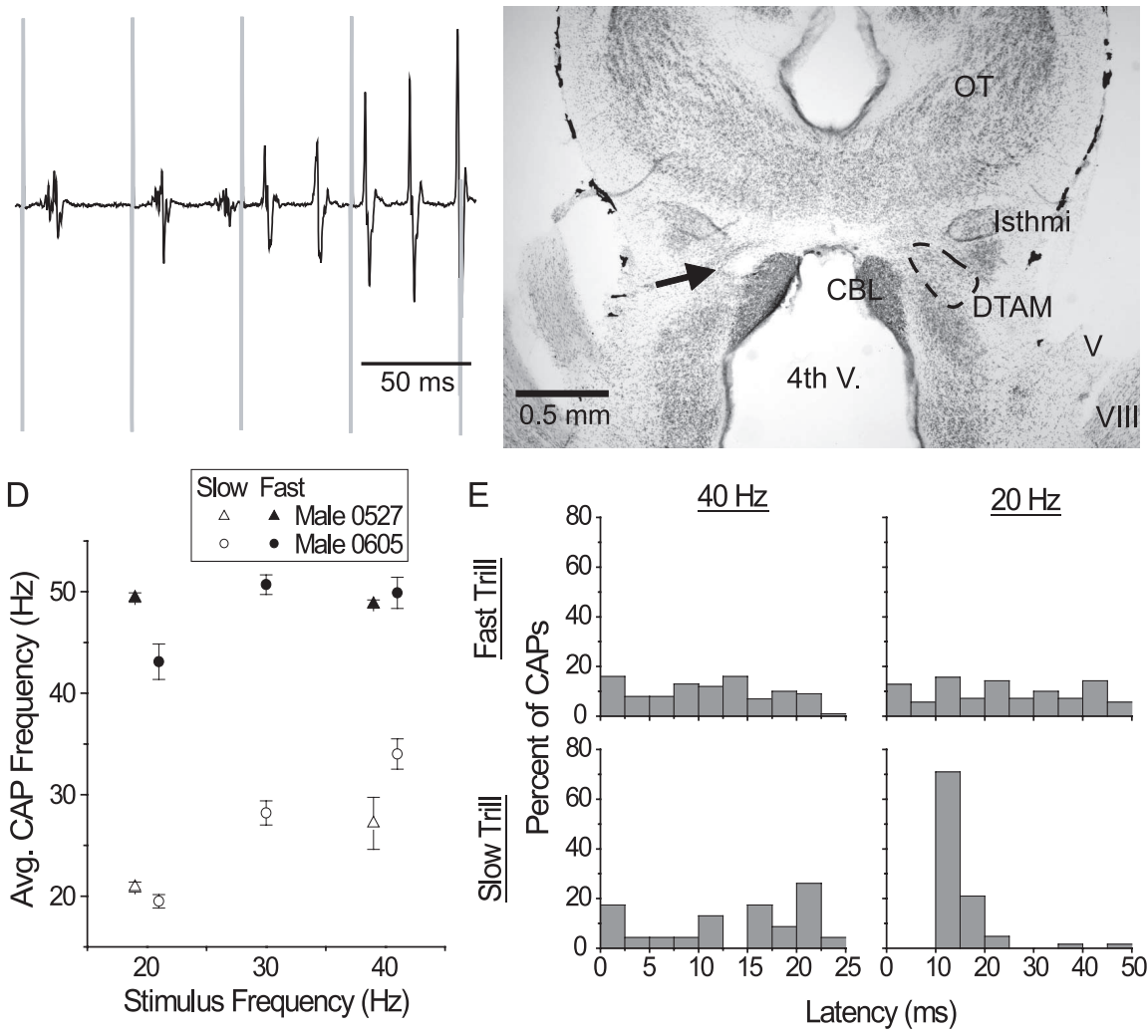

Figure 7. Electrical stimulation of DTAM can induce fictive advertisement call. $A$, A sample trace: continuous stimulation to DTAM at $20 \mathrm{~Hz}$ produced two bouts of fictive advertisement call. Stimulus artifact is gray, and nerve response is black. $\boldsymbol{B}$, Enlargement of gray boxed region in $A$ showing characteristic CAP shapes for slow and fast trill. C, A cresyl violet-stained horizontal section shows the lesion (arrow on left) at the rostral end of DTAM (location marked with dashed line on right). Fourth V, Fourth ventricle; CBL, cerebellum; Isthmi, nucleus isthmi; $0 \mathrm{~T}$, optic tectum; $\mathrm{V}$, nerve V; VIII, nerve VIII. $D$, Average CAP rate during slow and fast trill in response to three stimulus frequencies in two brains. Slow trill rate increases with increasing stimulus frequency, whereas fast trill does not change. Error bars are SEM. E, Frequency histograms show the distribution of CAP latencies from preceding stimuli for fast (top row) and slow (bottom row) trill, in response to stimuli at $40 \mathrm{~Hz}$ (left column) and $20 \mathrm{~Hz}$ (right column). Only slow trill with $20 \mathrm{~Hz}$ stimulation shows a strong relationship between stimulus and CAP timing.

mogenesis (Bass and Baker, 1997). In another frog species, Rana pipiens, the pretrigeminal nucleus (anatomically homologous to DTAM) is a component of the vocal CPG (Schmidt, 1976, 1992). Based on our results and homology to Rana pipiens, we propose that DTAM is both serotonin sensitive and a component of the vocal CPG in male and female Xenopus.

The vocal CPG could be composed of a single group of pacemaker cells, in DTAM or elsewhere, that drive the rest of the vocal-motor circuit. Alternatively, the CPG could consist of multiple populations of neurons that generate vocal patterns through dynamic interactions. Evidence may already favor a network CPG. Our experiments demonstrated that the slow and fast trills 
A

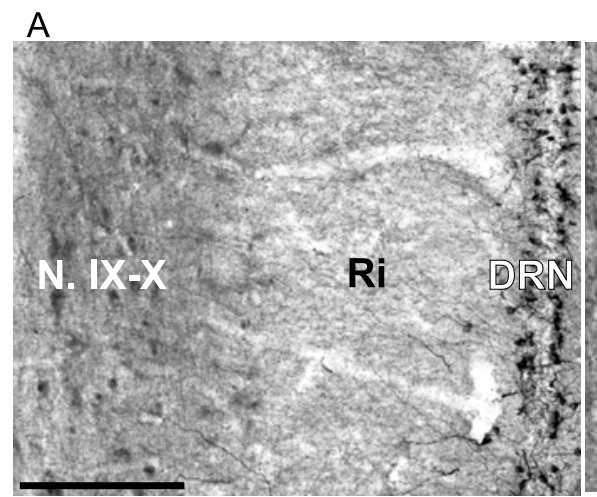

B

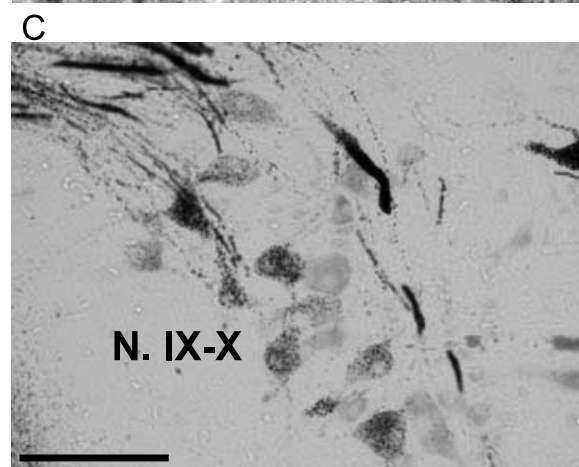

E

4th V.
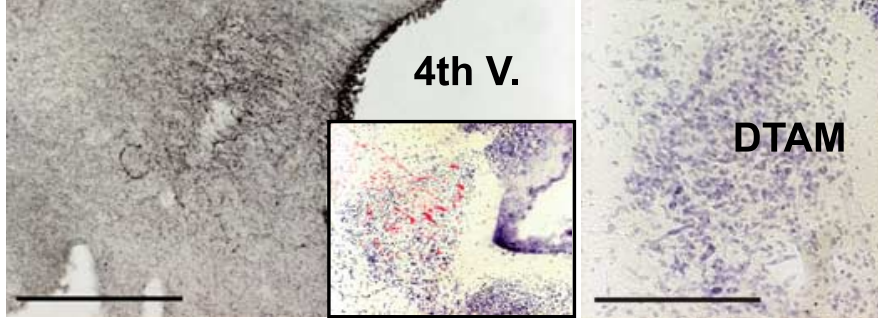

Figure 8. Endogenous serotonergic inputs to motor and premotor nuclei. $A, B$, Serotonergic neurons (black) are located along the midline of the brainstem in the dorsal raphe nucleus (DRN) and project laterally through the reticular formation (Ri) and into the laryngeal motor nucleus (N. IX-X). Arrowheads in $\boldsymbol{B}$ indicate varicose serotonergic fibers in the laryngeal motor nucleus. Sections in $\boldsymbol{A}-\boldsymbol{D}$ are horizontal, medial is to the right, and rostral is to the top. Scale bars: $A, 500 \mu \mathrm{m} ; \boldsymbol{B}, 250 \mu \mathrm{m}$. C, Backfill of nerve IX-X with the transneuronal tracer WGA-HRP (punctate black stain) resulted in filled motoneuron axons (top left) and cell bodies. Counterstained with cresyl violet (light gray). Scale bar, $100 \mu \mathrm{m} . \boldsymbol{D}$, Secondary label with transneuronal tracer was found in the DRN medial to laryngeal motor nucleus (arrowheads). Scale bar, $100 \mu \mathrm{m}$. $E$, Serotonergic fibers are found in the region of DTAM in the midbrain, lateral to the fourth ventricle (fourth V.) and ventral to the cerebellum (CBL). $\boldsymbol{F}$, The location of DTAM was confirmed with Nissl stain of an adjacent tissue section of $\boldsymbol{E}$ and further by back-labeling DTAM neurons with rhodamine dextran/ and overlaying with the adjacent Nissl-stained section ( $\boldsymbol{E}$, inset). Scale bars: $\boldsymbol{E}, \boldsymbol{F}, 500 \mu \mathrm{m}$.

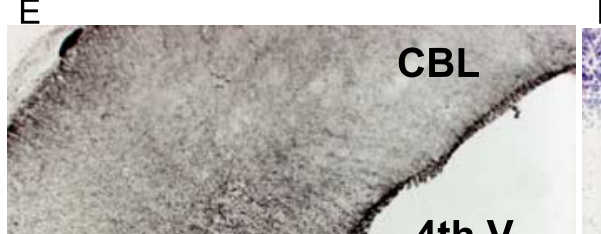

inferior reticular formations, and interneurons in nucleus IX-X are all candidates for participating in the CPG. Because both the reticular formation and nucleus IX-X were intact in the final condition of our transection experiments when fictive vocalizations were abolished, it seems unlikely that either nucleus, alone or in combination, is sufficient to serve as the CPG for any of the vocal patterns observed here. However, the possibility remains that DTAM is solely the site of action of 5-HT, triggering a more caudal CPG; future experiments will resolve this question.

\section{Sex-specific rhythmogenesis}

In this study, we found that 5-HT effectively evoked two types of fictive vocal behavior: the testosterone-dependent male advertisement call and the sexually undifferentiated release call. Although throughout this study we referred to "the" vocal CPG, the coexistence of these two very different rhythmic patters in male and $\mathrm{T}$-female brains may indicate that there are in fact two (or more) distinct or overlapping CPGs responsible for Xenopus vocal behavior. How does testosterone provide this gain-of-function, altering or adding to the undifferentiated CPG to produce male call patterns?

A variety of evidence suggests that male and female calling patterns are generated by the same neural circuit elements. Application of the same neurotransmitter effectively elicited fictive behavior in both sexes, suggesting a common role for 5-HT. Anatomically, the vocal pathways are composed of the same nuclei with similar patterns of connectivity, although there are differences in neuronal number and size (Kelley et al., 1988; Brahic and Kelley, 2003; Potter et al., 2005). Results of the transection experiments were the same in both sexes, suggesting that rhythmogenesis occurs at the same location. Furthermore, because testosterone does not appear to induce neurogenesis, at least not in nucleus IX-X (Kay et al., 1999), it is unof the male advertisement call could be altered independently (transection experiments perturbed the length and amplitude of the fast trill exclusively; the rate of electrical stimulation of DTAM affected the rate of slow trill only). This suggests that separate mechanisms, possibly in separate neuronal populations, establish the characteristics of the slow and fast trills.

Schmidt (1992) proposed a two-part CPG in Rana pipiens, which required network interactions between the pretrigeminal nucleus and a region coextensive with the nucleus IX-X to generate patterned vocalizations. Although vocal behavior is significantly different in Rana (a terrestrial frog that coordinates vocal and respiratory muscles), a similar network CPG could exist in Xenopus. Based on anatomic connectivity, DTAM, medial and likely that masculinization results in new circuit elements; rather, intrinsic properties of existing vocal neurons are probably remodeled, and their connections are reshaped. Specifically, testosterone may do one or more of the following, leading to the creation of a novel pattern generator or the unmasking of a latent one: alter the effect of 5-HT on vocal neurons (e.g., through changes in 5-HT receptor expression), alter membrane properties to allow faster firing patterns, or alter neuronal connectivity creating novel networks.

Neurons throughout the vocal pathway in both sexes, including those in nucleus IX-X and DTAM, express androgen receptors and could be direct targets of androgen action (Kelley et al., 1975; Kelley, 1981; Perez et al., 1996). We previously demon- 
strated that laryngeal motoneuron soma size and physiological properties masculinize with testosterone exposure (Yamaguchi et al., 2003, 2004; Oberlander et al., 2004; Potter et al., 2005). The ratio of soma to dendrite surface area is relatively constant in frog motoneurons (Birinyi et al., 1992), suggesting that the testosterone-induced increase in female motoneuron soma size may be accompanied by increased dendritic arborization and possibly the formation of novel synaptic connections (but see Kelley et al., 1988). Testosterone-induced morphological and physiological changes have yet to be examined in premotor nuclei but are likely and may be responsible for sex-specific rhythmogenesis.

Steroid hormones have been shown to modify the function of sex-specific CPGs in other species. In weakly electric fish, Apteronotus, the male CPG produces higher-frequency output than the female CPG; rates of electric organ discharge in intact fish and firing rates of pacemaker and motoneurons were raised by chronic androgen treatment and lowered by estrogen treatment (Schaefer and Zakon, 1996). Similar effects were seen in many weakly electric fish (Zakon, 2000). Additionally, in the midshipman fish, the duration of fictive vocal patterns produced by territorial males are modulated by short-term exposure to 11ketotestosterone or estradiol (Remage-Healey and Bass, 2004, 2006).

\section{Context-specific rhythmogenesis}

Xenopus produce different call types based on social context (Kelley and Tobias, 1998; Tobias et al., 2004). Pattern generator systems often produce multiple rhythms, corresponding to different behaviors, which are activated in a context-specific manner. Sensory or hormonal cues activate different CPGs or alter CPG activity to achieve different rhythms. This can be achieved through a variety of mechanisms, including the activation of different populations of neurons or the release of different neuromodulators (Katz, 1995; Marder and Bucher, 2001).

In Xenopus, combinations of sensory stimuli (such as the detection of potential mates, clasping, or being clasped) and hormonal cues (including testosterone and estrogen) elicit different call types in vivo. Thus, different endogenous brain states as well as different exogenous treatments (such as application of neuromodulators or stimulation of different brain areas) are likely to elicit different call types in vitro. We observed only a subset of calls within the male and female repertoires as fictive vocalizations, but call types seemed consistent with the hormonal state of the animal before the brain was removed. Namely, males, which were sexually receptive, produced fictive versions of calls associated with sexual receptivity in vivo: advertisement and amplectant calls. Females were not sexually receptive and produced release call, associated with unreceptive females in vivo.

Alternatively, the methods used here to stimulate the CPG might bias the production of certain patterns over others. Applying different neuromodulators, such as dopamine or norepinephrine, might induce different fictive patterns. Future work investigating the circuit and cellular mechanisms of pattern generation will yield more information about how the Xenopus vocal system generates different rhythms.

\section{References}

Bass AH, Baker R (1990) Sexual dimorphisms in the vocal control system of a teleost fish: morphology of physiologically identified neurons. J Neurobiol 21:1155-1168.

Bass AH, Baker R (1997) Phenotypic specification of hindbrain rhombomeres and the origins of rhythmic circuits in vertebrates. Brain Behav Evol 50 [Suppl 1]:3-16.
Birinyi A, Antal M, Wolf E, Szekely G (1992) The extent of the dendritic tree and the number of synapses in the frog motoneuron. Eur J Neurosci 4:1003-1012.

Brahic CJ, Kelley DB (2003) Vocal circuitry in Xenopus laevis: telencephalon to laryngeal motor neurons. J Comp Neurol 464:115-130.

Cowley KC, Schmidt BJ (1994) A comparison of motor patterns induced by $N$-methyl-D-aspartate, acetylcholine and serotonin in the in vitro neonatal rat spinal cord. Neurosci Lett 171:147-150.

Grillner S, Ekeberg O, El Manira A, Lansner A, Parker D, Tegner J, Wallen P (1998) Intrinsic function of a neuronal network: a vertebrate central pattern generator. Brain Res Brain Res Rev 26:184-197.

Hannigan P, Kelley DB (1986) Androgen-induced alterations in vocalizations of female Xenopus laevis: modifiability and constraints. J Comp Physiol A Neuroethol Sens Neural Behav Physiol 158:517-527.

Jacobs BL, Fornal CA (1997) Serotonin and motor activity. Curr Opin Neurobiol 7:820-825.

Katz PS (1995) Intrinsic and extrinsic neuromodulation of motor circuits. Curr Opin Neurobiol 5:799-808.

Kay JN, Hannigan P, Kelley DB (1999) Trophic effects of androgen: development and hormonal regulation of neuron number in a sexually dimorphic vocal motor nucleus. J Neurobiol 40:375-385.

Kelley DB (1981) Locations of androgen-concentrating cells in the brain of Xenopus laevis: autoradiography with $3 \mathrm{H}$-dihydrotestosterone. J Comp Neurol 199:221-231.

Kelley DB, Tobias ML (1998) The vocal repertoires of Xenopus laevis. In: Neuronal mechanisms of communication (Hauser M, Konishi M, eds), pp 9-35. Cambridge, MA: MIT.

Kelley DB, Morrell JI, Pfaff DW (1975) Autoradiographic localization of hormone-concentrating cells in the brain of an amphibian, Xenopus laevis. I. Testosterone. J Comp Neurol 164:47-59.

Kelley DB, Fenstemaker S, Hannigan P, Shih S (1988) Sex differences in the motor nucleus of cranial nerve IX-X in Xenopus laevis: a quantitative Golgi study. J Neurobiol 19:413-429.

Marder E, Bucher D (2001) Central pattern generators and the control of rhythmic movements. Curr Biol 11:R986-R996.

Oberlander JG, Bose TO, Potter KA, Smith S, Yamaguchi A (2004) Sexual differentiation of vocal motoneurons in development of Xenopus laevis. Soc Neurosci Abstr 30:672.6.

Olucha F, Martinez-Garcia F, Lopez-Garcia C (1985) A new stabilizing agent for the tetramethyl benzidine (TMB) reaction product in the histochemical detection of horseradish peroxidase (HRP). J Neurosci Methods 13:131-138.

Perez J, Cohen MA, Kelley DB (1996) Androgen receptor mRNA expression in Xenopus laevis CNS: sexual dimorphism and regulation in laryngeal motor nucleus. J Neurobiol 30:556-568.

Potter KA, Bose TO, Yamaguchi A (2005) Androgen-induced vocal transformation in adult female African clawed frogs. J Neurophysiol 94:415-428.

Remage-Healey L, Bass AH (2004) Rapid, hierarchical modulation of vocal patterning by steroid hormones. J Neurosci 24:5892-5900.

Remage-Healey L, Bass AH (2006) A rapid neuromodulatory role for steroid hormones in the control of reproductive behavior. Brain Res 1126:27-35.

Rhodes H, Yamaguchi A (2005) $I_{\mathrm{h}}$ regulates neuronal excitability in the vocal-motor system of male Xenopus. Soc Neurosci Abstr 31:612.9.

Sassoon D, Segil N, Kelley D (1986) Androgen-induced myogenesis and chondrogenesis in the larynx of Xenopus laevis. Dev Biol 113:135-140.

Schaefer J, Zakon HH (1996) Opposing actions of androgen and estrogen on in vitro firing frequency of neuronal oscillators in the electromotor system. J Neurosci 16:2860-2868.

Schmidt RS (1976) Neural correlates of frog calling: isolated brain stem. J Comp Physiol A Neuroethol Sens Neural Behav Physiol 108:99-113.

Schmidt RS (1992) Neural correlates of frog calling: production by two semi-independent generators. Behav Brain Res 50:17-30.

Simpson HB, Tobias ML, Kelley DB (1986) Origin and identification of fibers in the cranial nerve IX-X complex of Xenopus laevis: Lucifer Yellow backfills in vitro. J Comp Neurol 244:430-444.

Thompson KJ (1986) Oviposition digging in the grasshopper. I. Functional anatomy and the motor programme. J Exp Biol 122:387-411.

Tobias ML, Barnard C, O’Hagan R, Horng SH, Rand M, Kelley DB (2004) Vocal communication between male Xenopus laevis. Anim Behav 67:353-365. 
Wetzel DM, Haerter UL, Kelley DB (1985) A proposed neural pathway for vocalization in South African clawed frogs, Xenopus laevis. J Comp Physiol A Neuroethol Sens Neural Behav Physiol 157:749-761.

Wilson DM (1961) The central nervous control of flight in a locust. J Exp Biol 38:471-490.

Wyman RJ (1976) Neurophysiology of the motor output pattern generator for breathing. Fed Proc 35:2013-2023.

Yager DD (1992) A unique sound production mechanism in the pipid anuran Xenopus borealis. Zoo J Linn Soc 104:351-375.

Yamaguchi A, Kelley DB (2000) Generating sexually differentiated vocal patterns: laryngeal nerve and EMG recordings from vocalizing male and female African clawed frogs (Xenopus laevis). J Neurosci 20:1559-1567.
Yamaguchi A, Kaczmarek LK, Kelley DB (2003) Functional specialization of male and female vocal motoneurons. J Neurosci 23:11568-11576.

Yamaguchi A, Potter KA, Bose TO (2004) Androgen-induced vocal masculinization in African clawed frogs. Soc Neurosci Abstr 30:334.11.

Zakon HH (2000) Sex steroids and weakly electric fish: a model system for activational mechanisms of hormone action. In: Sexual differentiation of the brain (Matsumoto A, ed), pp 95-112. Boca Raton, FL: CRC.

Zornik EJ (2006) Regulating breathing and calling in an aquatic frog: neuronal networks in the Xenopus laevis hindbrain. $\mathrm{PhD}$ thesis, Columbia University.

Zornik EJ, Kelley DB (2007) Breathing and calling: neuronal networks in the Xenopus laevis hindbrain. J Comp Neurol 501:303-315. 